\title{
Unité proposée basée sur l'approche actionnelle pour le développement de quelques compétences professionnelles chez les futurs-enseignants de la langue française
}

\author{
Préparé par : \\ Dr. Manal Mounir Abbas \\ Maître de conférences de Curricula et de Méthodologie du FLE \\ Dr_mnalMoner_2005@yahoo.com
}


Unité proposée basée sur l'approche actionnelle pour le développement de quelques compétences professionnelles

Dr. Manal Mounir Abbas

\title{
Unité proposée basée sur l'approche actionnelle pour le développement de quelques compétences professionnelles chez les futurs-enseignants de la langue française
}

\author{
Préparé par: \\ Dr. Manal Mounir Abbas \\ Professeur de curriculum et méthodes d'enseignement Français langue \\ Faculté des filles-Ain Shams University \\ Dr_mnalMoner_2005@yahoo.com
}

\section{Sommaire :}

Dans cette recherche, la chercheuse étudie l'effet d'une unité proposée basée sur l'approche actionnelle sur quelques compétences professionnelles chez les futurs enseignants de la langue française.La recherche actuelle essaie de répondre aux questions suivantes : Quelles sont les sous-compétences de gérer une classe nécessaires pour les enseignants de la langue française ? Quelle est l'unité proposée à la lueur de l'approche actionnelle pour développer ces compétences ? Quel est le degré d'efficacité de cette unité ?Les résultats de la recherche mettent en valeur l'efficacité de cette unité proposée.

Mots-clés : unité proposée, compétences professionnelles, approche actionnelle, futurs-enseignants, langue française 


\title{
الوحدة المقترحة استتادا إلى نهج العمل لتطوير بعض المهار ات

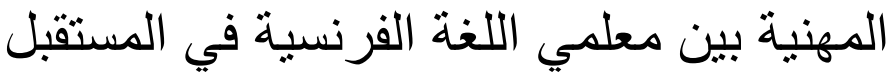

\author{
إعداد

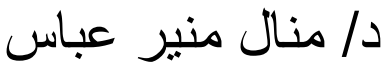 \\ مدرس المناهج وطرق تلدريس اللغة الفرنسية

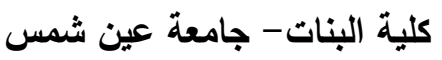 \\ Dr_mnalMoner_2005@yahoo.com
}

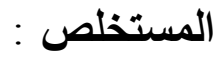

في هذا البحث ، يدرس الباحث تاثير الوحدة المقترحة استتادا إلى نهج العمل علي بعض المهارات المهنية بين معلمي اللغة الفرنسية في المستقبل ـ البحث الحالي يحاول الاجابه علي الاسئله الثالية: ما هي المهارات الفرعية لأداره الصف اللازم للمعلمين من اللغة الفرنسية ؟ ما هي الوحدة المقترحة في ضوء نهج العمل لنطوير هذه المهارات ؟ ما مدي فعاليه هذه الوحدة؟ وتشلط ننائج البحث الضوء علي فعاليه هذه الوحدة المقترحة. الكلمات المفتاحية : الوحدة المقترحة ، المهارات المهنية ، نهج العمل ، معلموا المستقبل ، اللغة

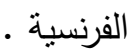




\section{Introduction}

Les compétences professionnelles de l'enseignant de la langue française sont nécessaires et pertinentes dans la formation initiale et la formation continue.

Les compétences professionnelles constituent une base essentielle pour tout enseignant de la langue française, surtout au début de son travail en classe.

Ces compétences professionnelles aident tout enseignant à détecter pourquoi ses élèves ont des difficultés à apprendre, ainsi que comment rendre leur apprentissage plus motivant.

Parmi ces compétences professionnelles qui causent des difficultés, citons celle du comment planifier et comment organiser les activités de l'enseignement de la langue française.

Ces problèmes sont mis en relief par quelques études antérieures, comme celle de Kounin (1970), celle de Yinger (1977) et celle de Struyk (1991).

Grâce à l'expérience personnelle de la chercheuse, en tant que formatrice des enseignants de la langue française, aussi bien que sa surveillance au stage pratique dans les écoles, la chercheuse constate qu'il existe quelques compétences professionnelles causant des difficultés aux futursenseignants.

La chercheuse a effectué une enquête consistant en un suivi d'un échantillon de 37 enseignants et enseignantes de secondaire, ceux-ci indiquent quels sont les objectifs les plus difficiles à atteindre pour eux. Une des conclusions est que "Faire travailler des élèves de niveaux hétérogènes et intéresser les moins motivés restent des difficultés, que n'ont pas toujours surmontées les enseignants ». Les plus grands besoins de ces enseignants sont les connaissances sur les façons de gérer les classes. De manière générale, on peut donc penser que, relativement à la plupart de ses aspects, la compétence de la gestion de classe ne découle pas purement et simplement de l'expérience. Malgré les efforts accomplis pour mettre en place une formation à l'acquisition de la compétence à gérer une classe, il n'en demeure pas moins qu'au long d'une carrière, un enseignant, même d'expérience, doit continuellement rajuster ses acquis en fonction de chaque nouvelle situation d'enseignement-apprentissage.

Le concept de gestion de classe s'est grandement transformé au cours des trois dernières décennies dans les recherches. Sa signification, réservée au départ à l'ordre et à la discipline dans la classe (McQueen, 1992; Burden, 1995), s'est peu à peu élargie pour englober maintenant l'ensemble des actes réfléchis, séquentiels et simultanés qu'effectuent les enseignants pour établir et maintenir un bon climat de travail et un environnement 
favorables à l'apprentissage (Archambault et Chouinard, 2003; Bowen, Desbiens, Gendron et Bélanger, 2005).

Le concept de gestion de classe renvoie désormais à tout ce qui préside à la planification et à l'organisation des situations d'enseignementapprentissage.

Des recherches empiriques sur cette question ont fait apparaître d'autres éléments cruciaux. Elles ont permis d'identifier des pertes du temps réel d'apprentissage liées à des répétitions de comportements et de consignes au début et à la fin d'une leçon et lors des transitions dans le déroulement d'une leçon (Arlin, 1979; Leinhardt, Weidman et Hammond, 1987; Jones, 1987; Brophy, 1988; Charlier, 1989; Burden, 1995; Yorke, 1998).

La gestion de la classe, rarement appliquée, constitue une référence majeure pour les enseignants francophones. Elle est à l'arrière-plan de la pensée enseignante, comme une sorte d'idéal ou de mauvaise conscience.

L'étendue du concept de gestion de classe amène à la considérer comme une des compétences majeures nécessaires en début de carrière, une compétence indispensable à l'établissement de la confiance en soi de l'enseignant et de son identité professionnelle.

L'importance de la gestion de classe, surtout pour les enseignants débutants, s'explique par le fait que si cette compétence n'est pas maîtrisée, il leur sera difficile d'acquérir les autres compétences nécessaires pour enseigner. Un enseignant débutant qui, en début d'année, ne réussit pas à instaurer ni à maintenir des routines relatives aux déplacements en classe et au bavardage, à orienter les élèves dans les étapes du déroulement d'une leçon ou à communiquer clairement les consignes pour accomplir une tâche, fera perdre beaucoup de temps et d'énergie à ses élèves. Ces nuisances à l'apprentissage découragent les élèves. De son côté, l'enseignant ne comprend pas pourquoi ses élèves n'apprennent pas. Son insatisfaction l'empêche de planifier des situations d'enseignement motivantes pour les élèves.

On pourrait évoquer bien d'autres exemples pour illustrer qu'un enseignant débutant qui ne maîtrise pas la compétence à gérer sa classe aura aussi des difficultés dans les autres facettes de son rôle, telles que celles de motivateur, de facilitateur, de négociateur ou d'organisateur.

En Egypte, malheureusement, la position traditionnelle typique consiste à dire que la compétence à gérer une classe s'acquiert par l'expérience, c'està-dire par essais et erreurs ou par imitation. Cette position mène alors à ne lui accorder pas une place dans les programmes de formation. 
Unité proposée basée sur l'approche actionnelle pour le développement de quelques compétences professionnelles

Dr. Manal Mounir Abbas

Traditionnellement aussi, il s'agit d'une compétence purement personnelle; l'inclure dans un programme de formation serait peine perdue.

Dans les deux cas, la conséquence est la même: elle aboutit à négliger cet aspect dans la formation.

En ce qui concerne l'acquisition de cette compétence, les résultats de plusieurs recherches, comme (Emmer et Stough, 2001; Murdock et Miller, 2003), fondés sur une population d'enseignants ayant des niveaux d'expérience très variés, montrent que la gestion de classe fait problème à tous et pas seulement aux débutants.

La question qui se pose alors est de savoir comment procéder pour aider les enseignants à développer leur compétence en ce domaine.

L'approche actionnelle est favorable pour apprendre et enseigner les langues vivantes. Cette approche constitue l'un des points clés de l'enseignement et de l'apprentissage des langues vivantes étrangères.

L'enseignant doit considérer l'apprenant comme acteur de ses apprentissages. Il le met en activité et introduit à cette occasion la langue et la culture comme instruments d'action et non seulement de communication. On ne communique plus seulement pour parler avec l'autre comme le proposait l'approche communicative des années 1980 mais on communique pour agir avec l'autre.

Cette approche représente un tournant dans l'enseignement et l'apprentissage des langues en ce sens qu'elle redéfinit le statut de l'apprenant et des tâches qu'il a à accomplir.

"La tâche est une visée actionnelle que l'acteur se représente comme devant parvenir à un résultat donné en fonction d'un problème à résoudre, d'une obligation à remplir, d'un but qu'on s'est fixé». (CECRL, p. 16).

L'apprenant apprendra par la réalisation de tâches, mais il est important de garder à l'esprit qu'il n'y a tâche que lorsque l'apprenant est motivé par l'action proposée. De plus, la tâche finale, qui constitue l'aboutissement d'une séquence pédagogique, pourra être réalisée si l'apprenant est entraîné tout au long de la séquence au moyen d'activités lui permettant d'acquérir les outils dont il aura besoin. En travaillant à la réalisation d'une tâche, la langue sera utilisée comme outil de communication et conférera ainsi une dimension authentique à la situation d'apprentissage.

Le moyen d'action est fondé sur la motivation des apprenants, suscité par l'aboutissement d'une réalisation concrète (dite tâche finale ou complexe). Elle induit un ensemble de tâches intermédiaires, dans lesquelles tous les 
Unité proposée basée sur l'approche actionnelle pour le développement de quelques compétences professionnelles

Dr. Manal Mounir Abbas

apprenants peuvent s'impliquer et jouer un rôle actif, qui peut varier en fonction de leurs besoins et intérêts. Il s'agit de donner du sens à l'apprentissage en mobilisant leur intérêt en les impliquant dans la résolution d'un problème qui fait naître le besoin de connaissances et donc le désir d'apprendre.

En suivant l'approche actionnelle, le cadre européen commun de référence propose des idées pour l'élaboration des unités didactiques, ce qui augmente l'autonomie chez les étudiants. 
Ainsi, proposer une unité, basée sur l'approche actionnelle, peut aider les futurs-enseignants à développer leurs connaissances, leurs aptitudes et leurs capacités à comment gérer leur classe de la langue française.

\section{Problématique de la recherche}

Le grand besoin des enseignants est l'acquisition des connaissances sur les façons de gérer leurs classes.

La recherche actuelle est censée répondre aux questions suivantes :

1. Quelles sont les compétences de la gestion de classe nécessaires pour les enseignants de FLE?

2. Quel est l'unité proposée à la lueur de l'approche actionnelle pour développer ces compétences de gestion de classe ?

3. Quel est le degré d'efficacité de cette unité proposée ?

\section{Hypothèse de la recherche}

Il existe une différence significative entre la moyenne des notes de l'échantillon dans le pré/post test en faveur du post test en ce qui concerne les compétences de la gestion de classe.

\section{Objectif et Importance de la recherche}

Présenter la manière de structurer ce savoir, la gestion en classe, pour le transmettre ou le faire acquérir au futur-enseignant et en même temps ajuster le savoir à ses priorités et à ses exigences.

\section{Outils de la recherche}

1. Une unité proposée pour le développement des compétences de la gestion de classe

2. Un test pour mesurer le degré de développement des compétences de la gestion de classe

3. Une grille d'observation des compétences de la gestion de classe

\section{Procédures de la recherche}

1. Etude des compétences de la gestion de classe

$>$ Un modèle théorique a été développé pour tenter d'une part de nommer les compétences de la gestion de classe pour ensuite en faire une meilleure analyse.

2. Etude de l'approche actionnelle

$>$ La nature de l'approche actionnelle. Le concept de la tâche. Les tâches et leur rôle dans la formation des enseignants. Le cycle de la tâche (la pré-tâche, la tâche et la post-tâche).

3. Elaboration des outils de la recherche

$>$ Une unité proposée basée sur l'approche actionnelle

$>$ Un pré-post test des compétences de la gestion de classe

$>$ Une grille d'observation des compétences de la gestion de classe

4. Application de l'expérimentation 
$>$ Choisir l'échantillon ( 1 groupe expérimental)

$>$ Appliquer le pré test

$>$ Appliquer l'unité proposée et la grille d'observation

$>$ Réappliquer le post test

5. Analyse et interprétation des résultats

\section{Terminologies de la recherche :}

\section{La gestion de classe :}

«Ensemble des pratiques éducatives auxquelles l'enseignant a recours afin d'établir, de maintenir et, au besoin, de rétablir dans la classe des conditions propices au développement des compétences des apprenants. » Archambault et Chouinard (2003)

Définition opérationnelle

La gestion de classe est un ensemble des actions qu'un enseignant (futurenseignant) conçoit, organise et réalise pour et avec ses apprenants, afin de les engager, de les soutenir, de les guider et de les faire progresser dans leur apprentissage et leur développement dans leur formation.

\section{L'approche actionnelle}

«L'approche actionnelle considère avant tout l'apprenant comme des acteurs sociaux ayant à accomplir des tâches (qui ne sont pas seulement langagières) dans des circonstances et un environnement donnés, à l'intérieur d'un domaine d'action particulier. »

Définition opérationnelle

Le Cadre européen commun de référence (CECR) L'approche actionnelle est une conception où l'étudiant (le futurenseignant) est un utilisateur qui accomplit des tâches: la classe est considérée comme le premier contexte situationnel, dans lequel cet étudiant aura des tâches à accomplir pour acquérir les compétences de la gestion de classe.

\section{Introduction}

En enseignement, la gestion de classe est un élément incontournable pour assurer un climat de classe propice à l'apprentissage. On remarque d'ailleurs chez du nouveau personnel enseignant des inconforts reliés à la gestion de classe. Par contre, les enseignants d'expérience peuvent aussi vivre des événements pouvant ébranler leur gestion de classe pourtant bien établie.

Soulignons que les difficultés reliées à la gestion de classe est la principale cause d'abandon de la profession enseignante en début de carrière et qu'un mauvais climat de classe peut avoir un impact négatif sur la persévérance scolaire des apprenants. 
La gestion de classe représente un défi pour plusieurs enseignants car elle est composée de plusieurs pratiques complexes qui doivent être actualisées souvent simultanément par l'enseignant. Tous n'ont pas la même approche. Ainsi, les enseignants se distinguent par leur profil personnel de gestion de classe, grandement influencé par leurs conceptions personnelles de l'enseignement et leurs représentations des besoins des élèves. Leur expérience, leur sexe, l'âge et le type d'élèves jouent également un grand rôle dans l'établissement de leurs différentes pratiques de gestion de classe.

Plusieurs raisons ont motivé les chercheurs et les praticiens à étudier la gestion de classe. Deux motifs majeurs de s'intéresser à ce sujet. D'abord, la gestion de classe fait partie du quotidien de milliers d'enseignants et d'élèves, et un temps considérable est absorbé par des tâches de gestion. Ensuite, les conclusions des nombreuses recherches indiquent l'existence de relations significatives entre la gestion de classe et une variété de retombées telles que la réussite, les attitudes et le comportement des élèves ainsi que la satisfaction au travail et l'efficacité des enseignants.

Plusieurs chercheurs sont venus corroborer l'importance de la gestion de classe sur la réussite éducative et scolaire (Wang, Haertel et Walberg, 1994) mais, comme le font remarquer Levin et Nolan (2000).

Une gestion de classe efficace est inséparable d'un enseignement efficace.

\section{Les compétences de la gestion de classe}

Un modèle théorique a été développé pour tenter d'une part de nommer les compétences de la gestion de classe pour ensuite en faire une meilleure analyse. Ce modèle se compose de neuf éléments.

\section{Gestion de classe: modèle théorique}

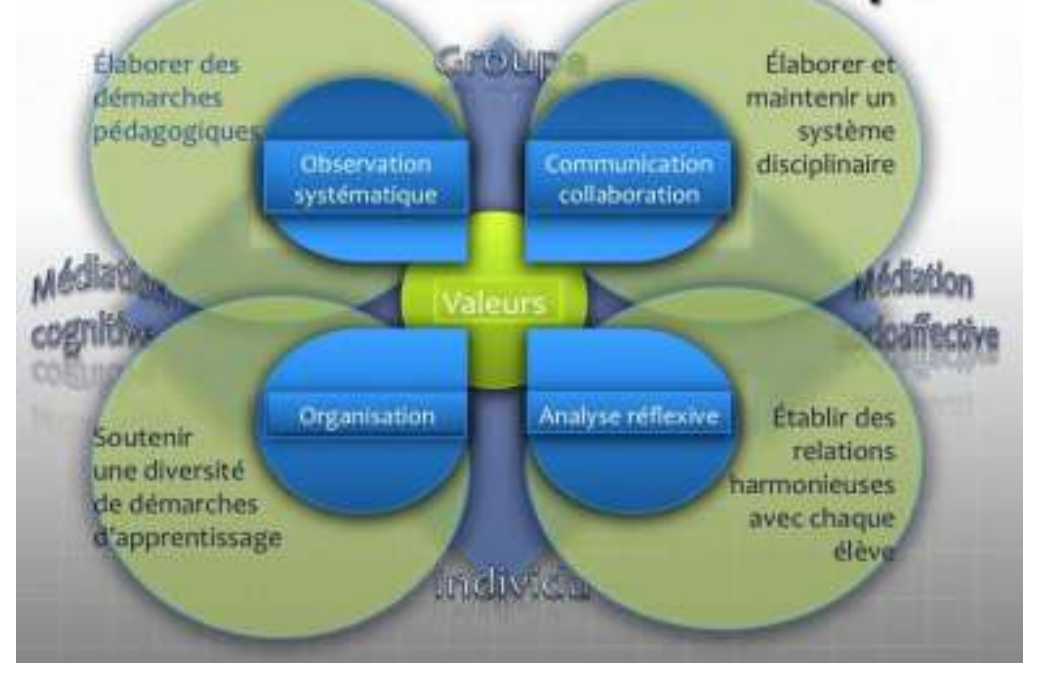


Figure 1 : Modèle théorique de gestion de classe (inspiré de Boutet, dans

Rousseau et Boutet (2003), adapté par Lessard et Schmidt, 2008)

$\mathrm{Au}$ milieu du modèle se retrouvent les valeurs. Ces valeurs influencent toutes les actions et les décisions de l'enseignant, notamment sur le plan des quatre éléments qui lui permettent de structurer son enseignement, soit l'organisation, l'observation systématique, la communication/la collaboration et finalement, l'analyse réflexive.

L'enseignement consiste à l'élaboration de démarches pédagogiques, mais un enseignant efficace se préoccupe aussi de soutenir une diversité de démarches d'apprentissage chez les élèves et de voir à ce que les besoins de ces élèves sur le plan socio-affectif soient comblés en établissant une relation harmonieuse avec chaque élève et en maintenant un système disciplinaire cohérent qui permet d'établir un climat propice à l'apprentissage.

1. Le centre du modèle de gestion : les valeurs

Les valeurs des enseignants guident l'ensemble de leurs décisions face à la gestion de classe et teintent toutes les dimensions de leur pratique et ont un lien direct avec les huit autres éléments du modèle théorique. Par leurs attitudes et comportements à l'égard des élèves, les enseignants laissent transparaître leurs valeurs associées non seulement à la réussite scolaire, mais aussi à l'effort et à la vie dans la société formée par les membres du groupe-classe. En tant que guide et modèle, l'enseignant peut transmettre ces mêmes valeurs à ses élèves et promouvoir différentes conduites et habiletés sociales qui en découlent afin de favoriser l'établissement de relations harmonieuses dans la classe (Richardson et Fallona, 2001). De plus, les valeurs, attitudes et comportements de l'enseignant peuvent être intégrés par les élèves qui désireront l'imiter (Archambault et Chouinard, 2003).

Pour la chercheuse, les valeurs que les futurs-enseignants ont à communiquer à leurs élèves sont : la gentillesse, l'équité, l'entraide, la coopération de l'apprentissage, l'empathie, le respect et l'autonomie.

2. L'organisation

Une bonne gestion de classe passe par l'ordre qui règne dans la classe. Plusieurs stratégies peuvent contribuer à y établir une organisation souple et efficace. Celles qui reviennent le plus fréquemment dans les écrits et qui ont été observées dans les pratiques de Calypso sont le partage des attentes, la gestion des transitions et les routines. En ayant recours à ces stratégies, l'enseignant développe un environnement structuré, ce qui 
permet aux élèves de se concentrer sur les apprentissages (Archambault et Chouinard, 2005; Johnson, Rice, Edgington et Williams, 2005).

La chercheuse estime que le futur-enseignant doit prendre le temps de réfléchir sur sa pratique et ses croyances personnelles afin d'identifier les conditions essentielles pour assurer la mise et place et le maintien d'une bonne organisation et d'un climat propice à l'apprentissage.

\section{L'observation systématique}

Que ce soit lors des leçons magistrales, du travail individuel ou en équipes, les enseignants efficaces supervisent l'ensemble de leurs élèves de façon à s'adapter à leurs besoins. Ils évaluent la compréhension et s'assurent de garder les élèves attentifs et engagés. En étant constamment à l'écoute et attentif à tout ce qui se passe dans la classe, ils récoltent les informations nécessaires pour maintenir un climat propice à l'apprentissage (Martineau et al., 1999).

La chercheuse déduit que l'observation systématique permet également au futur-enseignant de mieux connaître ses élèves de façon à ce qu'il puisse prendre des décisions adaptées aux caractéristiques et aux besoins de chacun.

\section{La communication et la collaboration}

En laissant une place aux élèves pour qu'ils s'expriment et en leur faisant pratiquer les habiletés de base pour transmettre un message (parler au «je», regarder et nommer la personne à laquelle je parle), l'enseignant montre qu'il accorde une place importante à la communication (Richardson et Fallona, 2001). Brown (2005) conseille à l'enseignant d'adopter une stratégie de communication active montre aux élèves qu'il les écoute vraiment à travers des signes verbaux et non verbaux et qu'il est sensible à ce qu'ils vivent. L'enseignant laisse aux élèves le temps de s'exprimer, il ne répond que lorsqu'ils ont terminé.

Par expérience, la chercheuse constate que les élèves aiment sentir que leur enseignant leur fait confiance et qu'il les considère suffisamment pour leur indiquer de façon transparente ce qu'il pense, qu'il soit sincère dans ses échanges avec eux.

\section{L'analyse réflexive}

Inviter les élèves à pousser plus loin leur raisonnement, à expliquer le processus qu'ils ont utilisé, leur compréhension les amène à développer leur pensée et à approfondir leurs connaissances. En étant sensibilisé aux conséquences de leurs actions, ils sont plus à même de faire des choix réfléchis et appropriés (Beyda et al., 2002; Richardson et Fallona, 2001). 
Pour la chercheuse, questionner c'est pour favoriser la réflexion : chez les futurs-enseignants cette catégorie est très représentative. Elle porte parfois sur les interventions didactiques et parfois sur les comportements des élèves.

\section{L'établissement de relations harmonieuses}

Pour fournir un enseignement individualisé et intervenir efficacement auprès des élèves, l'enseignant doit savoir ce qui caractérise chacun: ses forces, ses difficultés, ses qualités et ses intérêts (Bowen et al., 2005; Bru et al., 2002; Klem et Connell, 2004; Muller, 2001). Le fait d'établir une relation chaleureuse, caractérisée par l'implication émotionnelle, peu de dépendance et de conflits (Harme et Pianta, 2001) avec chacun des élèves favorise leur motivation, leur réussite et le développement d'un sentiment d'appartenance à l'école (Lapointe et Legault, 2004; Murdock et Miller, 2003).

Pour la recherche actuelle, la chercheuse tient à certaines stratégies qui permettent de développer de bonnes relations, notamment, d'adopter et de faire adopter aux futurs-enseignants des attitudes positives, de fournir des occasions aux futurs-enseignants de connaître le succès et de développer leurs compétences et de les encourager à consigner leur progrès.

7. L'élaboration et le maintien d'un système disciplinaire

Pour assurer un environnement propice à l'apprentissage, l'enseignant doit, dès la rentrée scolaire, expliquer aux élèves quelles sont ses attentes au regard de leur comportement et faire en sorte, tout au long de l'année, qu'ils répondent à ces attentes (Archambault et Chouinard, 2003; Rosenberg et Jackman, 2003).

La chercheuse estime un système de règles et de procédures qui favorise le maintien de l'ordre et de l'organisation en classe et qui fait en sorte que les futurs-enseignants peuvent se concentrer sur le développement de leurs compétences.

8. L'élaboration de démarches pédagogiques

Les situations d'enseignement-apprentissage constituent le cœur de la vie dans une classe. La préparation de celles-ci favorise leur bon déroulement, l'atteinte des objectifs de l'enseignant et l'engagement des élèves (Archambault et Chouinard, 2003; Bowen et al., 2005).

Pour favoriser l'engagement des futurs-enseignants, la chercheuse les incite à s'impliquer dans les tâches, à chercher les réponses à toutes les questions, même si ce ne sont pas eux qui y répondent et à jouer un rôle actif dans la construction de leurs apprentissages.

9. Le soutien d'une diversité de démarches d'apprentissage 
Dans le but de favoriser la réussite pour tous, l'enseignant doit idéalement adapter son enseignement aux différents styles d'apprentissage des élèves. Il accompagne les élèves pour les faire progresser à leur rythme et les amener à développer au maximum les compétences visées par le programme de formation (Archambault et Chouinard, 2003).

En amenant les futurs-enseignants à devenir autonomes, à prendre leurs propres décisions et en leur donnant du pouvoir dans la classe, la chercheuse leur permet de se réapproprier leur démarche d'apprentissage. Démontrer comment un enseignant arrive à établir un contexte dans lequel chaque élève peut atteindre son potentiel sur le plan de l'apprentissage et de son développement socio-affectif est très complexe.

Le modèle théorique de gestion de classe présenté démontre qu'un équilibre doit être atteint entre plusieurs tâches et fonctions pour qu'un enseignant arrive à aider les élèves à développer leurs compétences. L'enseignant doit se soucier à la fois du groupe, mais aussi des individus composant se groupe et il doit se soucier de leur développement cognitif et socio-affectif. Il doit établir et maintenir un climat propice à l'apprentissage tout en élaborant des démarches pédagogiques qui répondent aux divers besoins des apprenants. Son organisation et le recours à l'observation systématique, à une bonne communication et collaboration et finalement à l'analyse réflexive peuvent contribuer à rendre ce rôle un peu plus facile à gérer.

C'est pourquoi, la chercheuse pense à l'importance d'élaborer une unité proposée, comme un moyen de soutenir le travail du personnel enseignant et, par le fait même, la réussite des apprenants.

Le Cadre européen commun de référence (CECR) adopte une approche actionnelle qui vise à préparer les apprenants à être des acteurs à travers la réalisation de tâches réelles (effectives) ou proches de la vie réelle (virtuelle) en classe de langue étrangère. Cette nouvelle approche, qui commence petit à petit à remplacer les exercices et les activités traditionnels, offre de nouvelles perspectives à la formation des enseignants.

Il est important, pour notre recherche, de retenir qu'il y a tâche si l'action est motivée par un objectif ou un besoin, suscité par la situation d'apprentissage.

\section{La nature de l'approche actionnelle}

Selon le CECR, l'approche actionnelle est centrée sur la notion de tâche et elle "considère avant tout l'usager et l'apprenant comme des acteurs ayant à accomplir des tâches dans des circonstances et un 
Unité proposée basée sur l'approche actionnelle pour le développement de quelques compétences professionnelles

Dr. Manal Mounir Abbas

environnement donné, à l'intérieur d'un domaine d'action particulier ». (2005: 15)

Dans le cadre de cette approche, l'apprenant est un individu qui accomplit des tâches contextualisées à l'image du cadre social, professionnel, dans lequel il est ou sera inséré.

Dans cette approche, les activités de classe sont donc étroitement liées aux activités sociales que les apprenants sont ou peuvent être appelés à accomplir en société. De ces faits, dans le domaine de la formation des enseignants, on dit, plus facilement que l'apprenant a des tâches à accomplir et non pas des exercices à faire.

En effet, l'enseignant doit tenter d'importer en classe des situations propres au contexte social des apprenants et veiller à ce que les tâches réalisées soient transposables en contexte réel.

Par suite, l'enseignant est censé maîtriser finement les contextes sur lesquels reposent les tâches proposées aux apprenants. Et, naturellement, les apprenants sont censés se débrouiller dans ces contextes pour réaliser de façon optimale les tâches proposées.

Il est difficile de comprendre l'essence de l'approche actionnelle si on ne s'attarde pas sur la notion de "tâche" telle qu'elle est définie par le CECR. En outre, on peut difficilement cerner les fondements de cette approche si, à la lumière du CECR, on n'établit pas une différence entre "tâche" et "exercice".

D'après le CECR "Les tâches ou activités sont l'un des faits courants de la vie quotidienne dans les domaines personnel, public, éducationnel et professionnel. L'exécution d'une tâche par un individu suppose la mise en cuvre stratégique de compétences données, afin de mener à bien un ensemble d'actions finalisées dans un certain domaine avec un but défini et un produit particulier $\gg(2005: 15)$.

D'après la définition de tâche proposée par le CECR, l'apprenant (le futur-enseignant) est appelé à actualiser de façon stratégique un certain nombre de compétences devant une situation, où il agit de façon réfléchie pour atteindre un but déterminé.

Nous pouvons ainsi considérer que la tâche sort l'apprenant (le futurenseignant) de la dimension métalinguistique imposée par les traditionnels exercices. La tâche montre qu'en classe de langue, les activités réalisées sont avant tout des activités fondées sur l'interaction.

Notons enfin que la formation des enseignants fondé sur l'accomplissement de tâches favorise de toute évidence l'émergence de ce que nous appelons un apprenant réfléchi, c'est-à-dire un apprenant qui 
Unité proposée basée sur l'approche actionnelle pour le développement de quelques compétences professionnelles

Dr. Manal Mounir Abbas

réfléchit en action et sur son action, un apprenant qui développe des stratégies afin d'optimiser son développement professionnel.

Pécheur (2010), lors de son intervention au $7^{\mathrm{e}}$ congrès Panhellénique et International des Professeurs de Français à Athènes, schématise l'approche actionnelle comme suit :

\begin{tabular}{|llllll|}
\hline $\begin{array}{l}\text { Apprenant-utilisateur : } \\
\text { l'apprentissage) }\end{array}$ & acteur & social & (au centre & de \\
\hline
\end{tabular}

Tâches \begin{tabular}{lllll|}
$\begin{array}{l}\text { Acquisition de savoir-faire, savoir, savoir-être, savoir- } \\
\text { apprendre. }\end{array}$ \\
\hline
\end{tabular}

Contextes et situations

Schéma 1 : L'approche actionnelle selon Pécheur (2010)

L'intérêt de cette schématisation de l'approche est triple :

- passer de l'homme en tant qu'acteur réagissant à un acteur agissant ;

- l'interaction entre l'utilisateur et l'environnement est pensée comme tâche à accomplir ;

- ce schéma vaut autant pour l'utilisateur que pour l'apprenant.

Pour la chercheuse, le futur-enseignant est un utilisateur qui accomplit des tâches : la classe est considérée comme le premier milieu social ou le premier contexte situationnel dans lequel le futur-enseignant aura des tâches à accomplir.

\section{Qu'est-ce qu'une tâche ?}

Le concept de tâche n'est pas nouveau dans la didactique des langues ; c'est aux recherches et aux didacticiens anglo-saxons, comme Nunan (1989), Willis (1996) et Ellis (2003) que l'on doit l'approche actionnelle, fondée sur la réalisation des tâches Task-Based learning (TBL).

Le noyau central de cette approche est que les apprenants soient confrontés activement à la résolution d'un problème. Ensuite, cette notion a été largement reprise par le CECR.

Nunan définit la tâche de la manière suivante : «Une tâche est un plan de travail en classe qui implique les apprenants dans la compréhension, la manipulation, la production ou l'interaction » (1989: 10).

Ellis propose la définition suivante : "Une tâche est un plan de travail qui exige des apprenants qu'ils traitent de manière pragmatique dans le but de réaliser un résultat qui peut être évalué selon que le contenu des propositions a été réalisé de façon correcte ou appropriée. » (2003 : 16)

Le CECR définit la notion de tâche ainsi : "Toute visée actionnelle que l'acteur se présente comme devant parvenir à un résultat donné en fonction d'un problème à résoudre, d'une obligation à remplir, d'un but 
qu'on s'est fixé [...] Si l'on pose que les diverses dimensions ci-dessus soulignées se trouvent en interrelation, on pose aussi que tout acte d'enseignement d'une langue est concerné, en quelque manière, par chacune de ces dimensions: stratégies, tâches, textes, compétences individuelles, compétence langagière à communiquer, activités et domaines. » $(2005: 16)$

Cette définition adoptée par le CECR est très proche de celle formulée par Ellis, car on y retrouve la notion de résultat.

Ellis (2003: 1-35) identifie plusieurs dimensions, que l'on a adaptées à notre manière, pour définir une tâche :

- «La portée de la tâche »: elle permet de distinguer la fonction sémantique de l'exercice et pragmatique de la tâche.

- «L'approche à partir de laquelle la tâche est considérée »: elle permet de distinguer le canevas imaginé par l'enseignant du processus effectivement mis en place par les apprenants. Ceci relève de la distinction entre la tâche centrée sur le sens et celle centrée sur la forme.

- «L'authenticité de la tâche ». elle prône des tâches présentant deux types d'authenticité : Authenticité situationnelle, lorsque la tâche correspond à des actions de la vie réelle, et Authenticité interactionnelle, lorsque la tâche permet, aux apprenants la mise en œuvre de processus présents dans la vie réelle.

- "Les processus cognitifs mis en place lors de la réalisation d'une tâche » : la réalisation d'une tâche engage un certain nombre de processus cognitifs, comme par exemple la sélection, le raisonnement, la classification, etc.

- «Le résultat de la tâche »: il est nécessaire, ici, de distinguer entre le résultat et l'objectif de la tâche. Le résultat se réfère à ce que les apprenants sont arrivés à faire une fois la tâche terminée. Par contre, l'objectif se réfère aux propositions pédagogiques de la tâche.

Nous remarquons à travers ces définitions de la tâche qu'il est généralement convenu que les tâches doivent avoir des objectifs, réalisés par l'engagement des participants dans un comportement axé sur le but.

\section{Les tâches et leur rôle dans la formation des futurs-enseignants}

Selon Ellis, beaucoup de linguistes, de didacticiens et de spécialistes reconnaissent les avantages de l'introduction des tâches en classe de langues étrangères. Elles peuvent être introduites de deux manières (2003: 27) :

- soit on les introduit dans les méthodes traditionnelles d'enseignement (Task-supported language teaching) ; 
- soit on élabore l'ensemble du cursus autour des tâches (Taskbased language teaching)

Il est à souligner que dans les deux cas, l'utilisation des tâches dans la classe rend l'enseignement/apprentissage plus communicatif et interactif. En effet, les tâches développent chez les apprenants la capacité d'une utilisation dans de vraies situations liées au monde réel.

Dans une situation qui suppose l'utilisation de la langue étrangère en voie d'acquisition, les tâches peuvent avoir deux fonctions (2006:38) :

- une fonction interactionnelle qui sert à établir et à maintenir le contact. Elle doit donc impliquer une communication la plus réelle possible entre les apprenants ;

- une fonction transactionnelle servant à échanger de l'information entre les interlocuteurs.

Dans cette perspective, l'introduction des tâches en classe est justifiée dans la mesure où elle permet de créer de l'interaction par laquelle passe l'enseignement/apprentissage d'une langue étrangère, les tâches développent ces deux habilités chez les apprenants à travers la mise en œuvre de situations réelles.

Pour certains auteurs comme Nunan, les tâches sont placées au centre de toute conception du processus d'enseignement et d'apprentissage. Nunan (1989: 11) représente le statut qu'il donne à la tâche de la manière suivante :

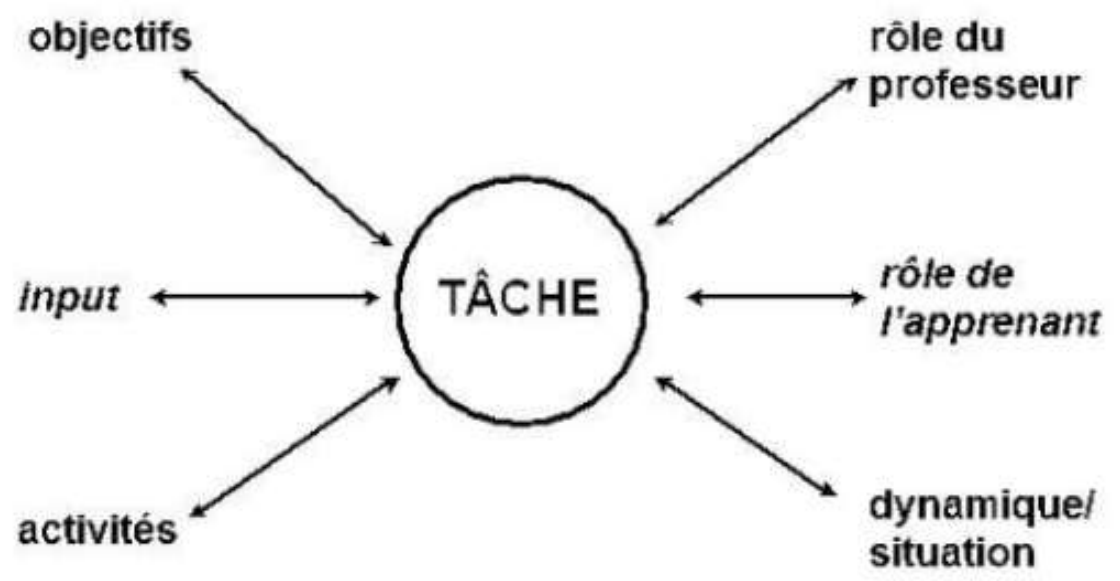

Schéma 2 : Le statut de la tâche selon Nunan (1989)

D'après ce schéma, nous remarquons que la conception d'une tâche exige la prise en considération de six paramètres : les objectifs, le support de 
départ (input), les activités que les apprenants doivent effectuer, les rôles respectifs de l'apprenant et de l'enseignant, et enfin la situation.

D'autres auteurs, comme Willis et Ellis ont ajouté un septième paramètre : le résultat, qui constitue pour les apprenants l'objectif de la tâche.

Le CECR considère l'approche actionnelle comme le centre de gravité de toute la conception du processus d'enseignement. Cette approche permet de repenser l'enseignement en tenant compte des besoins des apprenants et en motivant le travail par une production finale.

Springer affirme que «la tâche pédagogique constitue l'élément moteur d'une rénovation pédagogique de type actionnel $\gg(2009$ : 33).

Pour notre recherche, le recours aux tâches en classe doit s'appuyer aussi sur l'imaginaire et la créativité, ce qui facilite bien évidemment l'enseignement/l'apprentissage dans la mesure où les futurs-enseignants sont placés dans des situations proches du réel. Ces tâches simulées, bien qu'elles ne soient pas toujours identiques à la vie réelle, permettent aux futurs-enseignants de créer des interactions et des échanges.

\section{Le cycle de la tâche :}

La tâche s'offre à l'apprenant sous la forme d'un cycle de tâche : la prétâche, la tâche et la post-tâche.

- Durant la pré-tâche, l'apprenant est amené à mobiliser les schémas mentaux et les scénarios lui permettant de planifier la tâche au niveau de la macro-structure du contenu global, mais aussi de prévoir les éléments de la méso-structure au niveau du scénario et ceux de la micro-structure au niveau du matériau à mobiliser ou à acquérir, nécessaire à l'exécution de la tâche.

- Durant l'exécution de la tâche, la phase de négociation avec l'enseignant mettra au point les modalités et la stratégie lui permettant de réaliser son objectif et définira les critères et les indices lui permettant d'évaluer la qualité des processus et du produit envisagé.

- Après la réalisation de la tâche, l'apprenant la présente à son public. Cette phase de présentation sera suivie d'une évaluation par le groupe et d'une auto-évaluation. Il y aura donc des moments de réflexion permettant de faire le point sur les acquis et les processus ayant mené à ces acquis.

\section{Tableau 1 : Le cycle de la tâche}

\begin{tabular}{|c|c|c|}
\hline $\begin{array}{l}\text { La pré- } \\
\text { tâche }\end{array}$ & $\begin{array}{l}\text { orientation sur la } \\
\text { tâche }\end{array}$ & $\begin{array}{l}\text { lien avec la vie réelle et } \\
\text { mobilisation } \\
\text { connaissances }\end{array}$ \\
\hline
\end{tabular}


Unité proposée basée sur l'approche actionnelle pour le développement de quelques compétences professionnelles

Dr. Manal Mounir Abbas

\begin{tabular}{|c|c|c|}
\hline & Négociation & $\begin{array}{l}\text { négociation de la tâche et des } \\
\text { modalités d'exécution : qualité } \\
\text { requise du produit fini, gestion } \\
\text { du temps et de l'espace }\end{array}$ \\
\hline & Ressources & $\begin{array}{lll}\text { matériaux } & \text { requis, } & \text { aide } \\
\text { disponible } & & \\
\end{array}$ \\
\hline \multirow[t]{2}{*}{ La tâche } & Planification & démarche et plan \\
\hline & $\begin{array}{l}\text { exécution de la } \\
\text { tâche }\end{array}$ & exécution de la tâche \\
\hline \multirow[t]{3}{*}{$\begin{array}{l}\text { La post- } \\
\text { tâche }\end{array}$} & $\begin{array}{l}\text { présentation de la } \\
\text { tâche }\end{array}$ & présentation orale ou écrite \\
\hline & $\begin{array}{l}\text { réflexion et feed- } \\
\text { back }\end{array}$ & $\begin{array}{l}\text { évaluation, autoévaluation } \\
\text { énumération des acquis et } \\
\text { évaluation de la démarche }\end{array}$ \\
\hline & $\begin{array}{l}\text { structuration des } \\
\text { acquis }\end{array}$ & $\begin{array}{l}\text { cadres de référence, } \\
\text { schémas... replaçant les acquis } \\
\text { dans le contexte du système }\end{array}$ \\
\hline
\end{tabular}

L'approche actionnelle consiste en une compilation de micro-tâches d'apprentissage qui préparent les apprenants individuellement à réaliser une macro-tâche en collaboration, en guise de transfert.

En adoptant l'approche actionnelle, le CECR nous a apporté de nouvelles perspectives de conception et d'élaboration de cours. La mise en œuvre d'une tâche efficace renforce le processus d'apprentissage. Elle incite naturellement les futurs-enseignants à comprendre leurs propres processus d'apprentissage.

Comme on a pu le remarquer, l'approche actionnelle constitue une sorte de renouveau pédagogique pour les classes. L'introduction des tâches en classe peut s'avérer très utile pour les futurs-enseignants. Elle sert à activer l'apprentissage.

Par ailleurs, le recours aux tâches qui simulent des situations réelles préparent les futurs-enseignants à l'exécution des tâches réelles et donc à l'insertion dans la société où se passent les interactions.

\section{Les outils de la recherche et l'expérimentation}

Bien qu'il soit important de former les futurs-enseignants à la gestion responsable de la classe, la chercheuse a élaboré une unité basée sur l'approche actionnelle. 
Cette unité proposée constitue un document de référence pour la gestion d'une classe de la langue française tant au niveau du primaire qu'au niveau secondaire.

\section{L'unité proposée}

Cette unité (annexe 1) se compose de quatre dossiers, où le futurenseignant a des tâches à accomplir dans des activités, regroupées en 8 thèmes.

Dans chaque activité, la chercheuse présente une fiche pédagogique et une fiche technique.

La fiche pédagogique de chaque activité contient les sept points suivants :

- le titre de l'activité

- les objectifs spécifiques

- la durée

- le déroulement de l'activité

- les questions de synthèse.

Quant à la fiche technique, elle explicite des informations à communiquer au public cible.

Cette unité proposée vise, en général, à sensibiliser les futurs-enseignants sur l'importance de la classe dans l'œuvre éducative, à améliorer leur gestion dans son contexte actuel, à enrichir les méthodes et les techniques d'enseignement, à renforcer leurs compétences.

\section{Les objectifs de l'unité proposée \\ - Objectif général}

Permettre aux futurs-enseignants de développer des connaissances, des attitudes et des comportements susceptibles d'améliorer la gestion d'une classe de la langue française.

- Objectifs spécifiques

Permettre au futur-enseignant d'accomplir des tâches pour :

- Identifier, fabriquer et exploiter les supports pédagogiques

- Tenir et faire tenir correctement les documents pédagogiques

- Se servir correctement des méthodes d'organisation d'une classe

- Utiliser d'une manière appropriée les techniques d'organisation d'une classe

- Renforcer ses compétences sur les différents types d'évaluation

- Améliorer le climat de travail dans sa classe pour un apprentissage efficace

- Organiser des activités scolaires et parascolaires

- Planifier une séquence didactique selon la pédagogique par objectif

Les thèmes de l'unité proposée 
- La constitution d'un groupe

- Les techniques d'organisation d'une classe

- Le climat de travail en classe

- Les méthodes et les techniques d'enseignement

- Les supports pédagogiques

- Les documents pédagogiques

- L'évaluation pédagogique

- La pédagogie par objectifs

Les dossiers de l' unité proposée

L'unité proposée se compose de quatre dossiers. Chaque dossier traite deux thèmes. Chaque thème est mis en œuvre par des activités, où les futurs-enseignants ont des tâches à réaliser.

\section{Dossier 1}

Thème 1: La constitution d'un groupe

Activité 1 : Présentation des participants par l'Interview 2 à 2.

Activité 2 : Règles de fonctionnement

Thème 2 : Les techniques d'organisation d'une classe

Activité 1 : Composition d'une classe

Activité 2 : Techniques d'organisation d'une classe

Dossier 2

Thème 3 : Le climat de travail en classe

Activité 1 : Attitudes et comportements des enseignants

Activité 2 : Attitudes et comportements des élèves

Thème 4: Les méthodes et les techniques d'enseignement

Activité 1 : Méthodes et techniques actives et participatives

Activité 2 : Activités scolaires et parascolaires

Dossier 3

Thème 5 : Les supports pédagogiques

Activité 1 : Fabrication et exploitation du matériel didactique

Activité 2 : Usage adéquat des moyens d'enseignement

Thème 6 : Les documents pédagogiques

Activité 1 : Tenue des documents pédagogiques

Dossier 4

Thème 7: L'évaluation pédagogique

Activité 1 : Types d'évaluation pédagogique

Activité 2 : Moyens d'évaluation 
Thème 8 : La pédagogie par objectifs

Activité 1 : Formulation des objectifs pédagogiques

Activité 2 : Elaboration d'une fiche pédagogique

\section{Le pré-post test}

Le test (annexe 2) a pour objectifs de

$>$ mesurer le degré d'acquisition des connaissances, des attitudes et des comportements susceptibles d'améliorer la gestion d'une classe de la langue française.

$>$ mesurer le degré d'efficacité de l'unité proposée

Le pré-post test se compose de 10 tâches.

La note totale du test est 50 points.

\section{La grille d'observation}

Cette grille (annexe 3) sert à observer et ajuster les compétences de la gestion de classe durant la passation des activités de l'unité proposée. Elle se compose de 12 items valorisant le savoir-agir des futurs-enseignants.

\section{L'expérimentation}

Cette unité proposée a été expérimentée sur une période de trois mois avec les étudiantes de la $2^{\text {ème }}$ année pédagogique, à la faculté de JeunesFilles.

Les étapes de l'expérimentation:

$>$ La première séance est consacrée au test (pré-test) réalisé auprès de toutes les étudiantes (futurs-enseignantes).

$>\mathrm{La}$ chercheuse a exploité les dossiers didactiques composés de plusieurs activités, elle a utilisé la grille pour faire le suivi de quelques éléments.

$>$ Ensuite, elle a réappliqué le test (post-test) où elle évalue l'influence de l'unité proposée sur la gestion de classe.

$>$ Enfin, elle analyse et interprète les résultats.

\section{Remarques lors de l'expérimentation}

Le degré d'engagement des étudiantes dans les tâches dépend de la valeur qu'elles accordent à la tâche. Le rôle de la chercheuse a consisté à favoriser l'implication maximale des étudiantes en leur permettant de jouer un rôle actif dans leurs apprentissages et en leur faisant voir la pertinence des activités.

En proposant des activités stimulantes et riches de sens, la chercheuse a incité les étudiantes à s'engager dans la tâche. Elle s'assure par la suite de conserver l'attention et l'engagement des étudiantes tout au long de l'activité par diverses stratégies. 
Unité proposée basée sur l'approche actionnelle pour le développement de quelques compétences professionnelles

Dr. Manal Mounir Abbas

La stratégie fréquemment utilisée pour conserver l'attention des étudiantes est de leur poser diverses questions pour qu'elles cherchent la réponse dans leur tête. En réfléchissant pour trouver une solution, elles restent actives et engagées jusqu'à la fin.

L'évaluation formative (la grille) et sommative (le test) permettent à la chercheuse de se renseigner sur les apprentissages, les progrès et les difficultés des étudiantes. Par la suite, elle fait des retours sur la matière qui semble moins comprise afin de s'assurer que toutes développent les compétences visées par l'unité proposée .

\section{L'analyse et l'interprétation des résultats}

L'analyse porte sur les progrès réalisés entre le pré-post test, sur l'effet de l'utilisation de l'unité proposée sur la gestion de classe de la langue française chez l'échantillon.

Pour l'analyse statistique des résultats, la chercheuse a calculé la moyenne arithmétique des notes, l'écart type et la valeur de"T".

Tableau 2 : les résultats obtenus au pré-post test des compétences de la gestion de classe

\begin{tabular}{||c||c||c||c||c||c|c||}
\hline \hline Mesure & n & TN & TC & M & E & T \\
\hline Pré-test & \multirow{2}{*}{24} & 416 & 9052 & 17,3 & 8,8 & \multirow{2}{*}{9,37} \\
\cline { 1 - 5 } & 1022 & 45961 & 42,6 & 10,04 & \\
\hline
\end{tabular}

Le tableau ci-dessus indique le total des notes, le total des carrés de ces notes, la moyenne arithmétique, l'écart type et la valeur de " $\mathrm{T}$ " des étudiantes au pré-post test.

Notons que:

La différence entre la moyenne des notes des étudiantes au pré-post test est significative au niveau de 0.01 , en faveur du groupe après l'étude expérimentale. Ce qui valorise l'efficacité de l'unité proposée.

\section{Quelques remarques de la chercheuse}

La grille d'observation a aidé la chercheuse à dégager quelques remarques lors de l'observation des activités de l'unité proposée :

- Des actions sont menées en vue d'organiser l'environnement au niveau spatial et au niveau matériel ainsi que des actions sont réalisées en vue de changer cet environnement. 
- Des actions sont menées en relation à la dimension de l'organisation du groupe classe pour l'activité et des actions sont réalisées en vue de modifier cette organisation.

- Des actions sont menées en relation à la dimension sociale de la classe et à l'aménagement du code de vie. Elles consistent en communications destinées à mettre à la disposition de toutes les étudiantes les informations nécessaires.

- Des actions sont menées en relation à la dimension temporelle destinées à organiser les tâches au sein des moments didactiques en fonction du temps disponible et des actions de réorganisation de l'espace temps qui reste disponible.

- Des actions sont réalisées en fonction des comportements des étudiantes, des actions de contrôle, des sollicitations auprès des étudiantes à ce qu'elles réalisent elles-mêmes des actions nouvelles, qu'elles adoptent des comportements plus appropriés lors de la réalisation des activités.

- Des actions de régulation à caractère interactif sont réalisées. Elles ont pour but d'engager une relation particulière avec les étudiantes et sollicitent celles-ci à réagir, à modifier des comportements ou des attitudes adoptées.

\section{Conclusion}

Pour conclure notre recherche, il nous semble opportun de revenir à la question posée, qui ouvrait sur les actions de gestion de classe et la capacité des futurs-enseignants à entreprendre de telles actions.

La formalisation de certaines actions et l'explication du caractère contraignant des situations vécues par les futurs-enseignants sont des informations intéressantes mentionnées dans notre recherche.

La méthodologie, utilisée dans cette recherche, met en relief la construction de ces compétences de la gestion de classe pendant la formation initiale, en permettant au futur-enseignant d'analyser sa relation à une situation professionnelle qui s'est avérée pour lui problématique et dont il fait récit. 
Unité proposée basée sur l'approche actionnelle pour le développement de quelques compétences professionnelles

Dr. Manal Mounir Abbas

Elle s'accompagne aussi d'un développement de la capacité à structurer la réflexion.

En ce sens, la finalité de l'exploitation de l'unité proposée touche à un principe éthique, qui fonde la formation des futurs-enseignants : aider à la construction d'une identité professionnelle progressivement assurée et construire la compétence de gérer sa classe, en vue de faciliter l'adaptation aux interactions en classe ; aussi bien que donner l'occasion aux futurs-enseignants de se livrer, avec l'aide du formateur, à une réflexion sur les difficultés rencontrées en matière de gestion de classe.

Il s'agit là, d'une orientation dans le travail de formation qui prend toute sa place dans la formation initiale, parce qu'elle peut justement permettre l'ajustement des représentations des futurs-enseignants par rapport aux pratiques à envisager dans les situations difficiles, en l'outillant progressivement de catégories descriptives et d'analyse. 


\section{Bibliographie}

Archambault, J. et Chouinard., R. (2003). Vers une gestion éducative de la classe. 2e édition. Boucherville: Gaëtan Morin.

Arlin, M. (1979). Teacher transitions can disrupt time flow in classrooms. American Educational Research Journal, 16, A2-56.

Bérard, É. (2009). Les tâches dans l'enseignement du FLE : rapport à la réalité et dimension didactique. Le français dans le monde, $\mathrm{n}^{\circ}$ spécial «La perspective actionnelle et l'approche par les tâches en classe de langue », pp. 36-44.

Beyda, S. D., Zentall, S. S. et Ferko, D. J. K. (2002). The relationship between teacher practices and the task appropriate and social behavior of student with behavioural disorders. Behavioral Disorders. 27(3), 236-255.

Bowen, F., Desbiens, N., Gendron, M. et Bélanger, J. (2005). L'acquisition et le développement des habiletés sociales. In. N. Desbiens, C. Lanaris et L. Massé. (dir), Les troubles du comportement à l'école : Prévention, évaluation et intervention. (p. 213-227). Québec : Gaétan Morin.

Brophy, J.E.(1988). Education teachers about managing classrooms and students. Teaching and Teacher Education, 4, 1-18.

Bru, E., Stephens, P. et Torsheim, T. (2002). Students' perceptions of class management and reports of their own misbehavior. Journal of School Psychology. 40(4), 287- 307.

Burden, RR. (1995). Classroom management and discipline. Methods to facilitate cooperation and instruction. New York (NY) : Longman.

Charlier, E. (1989). Planifier un cours, c'est prendre des décisions. Bruxelles: De Boeck.

Chouinard, R. (1999). Enseignants débutants et pratiques de gestion de classe. Revue des sciences de l'éducation. 25(3), 497-514.

Chouinard, R., Plouffe, C. et Archambault, J. (2005). Le soutien de la motivation scolaire. In. N. Desbiens, C. Lanaris et L. Massé. (dir), Les troubles du comportement à l'école : Prévention, évaluation et intervention. (p. 261-270). Québec : Gaétan Morin.

Clark, CM. et Peterson, P.L. (1986). Teachers though processes. In M.C. Wittrock (dir.), Handbook of Research on Teaching (3e éd.) (p. 255-295). New York (NY) : Macmillan.

Conseil de l'Europe. (2001).Cadre européen commun de référence pour les langues, Paris, Didier.

Conseil de l'Europe. Guide pour les utilisateurs, Strasbourg. (2002) http://www.coe.int/t/dg4/portfolio/documents/guide-pour-utilisateursavril02.doc 
Conseil supérieur de l'éducation. (1995). Pour une gestion de la classe plus dynamique au secondaire.

Coste, D. (2009).Tâche, progression, curriculum. Le français dans le monde, $\mathrm{n}^{\circ}$ spécial «La perspective actionnelle et l'approche par les tâches en classe de langue », pp. 15-24.

DA COSTA, Paulo. (2002).Compétence de communication : Le pivot abstrait de la didactique des langues, Revista Kulonga (Revista das ciências da educação e estudos multidisciplinares), n², avril, p.43-60.

Desgagné, S. (1994). À propos de la «discipline de classe»:analyse du savoir professionnel d'enseignantes et enseignants expérimentés du secondaire en situation de parrainer des débutants. Thèse de doctorat. Québec : Université Laval.

Domalga-Zysk, E. (2006). The significance of adolescents' relationships with significant others and school failure. School Psychology International, 27(2), 232-247.

Doyle, W. (1986). Classroom organization and management. In M.C. Wittrock (dir.), Handbook of Research on Teaching (p. 392-431). New York (NY) : Macmillan.

Ellis, R. (2003).Task-based Language Learning and Teaching. Oxford: Oxford University Press.

Emmer, E. T. et Stough, L. M. (2001). Classroom management: A critical part of teacher educational psychology, with implications for teacher education. Educational Psychologist. 36(2), 103-112.

Fijalkow, J. (1996). Mauvais lecteurs, pourquoi? Paris : Presses universitaires de France.

Freinet, C. (1994). OEuvres pédagogiques. Paris: Seuil.

Hill, G. (1944). Teachers' instructional difficulties - A review of research. Journal of Educational Research, 37(8), 602-615.

Johnson, D. D., Rice, M. P., Edgington, W. D. et Williams, P. (2005). How to succeed in classroom. Kappa Delta Pi Record. 42(1), 28-32.

Jones, EH. (1987). Positive classroom discipline. New York (NY) : McGraw-Hill.

Kazeroni, A. (2004).La construction d'une tâche d'apprentissage d'une langue étrangère dans des environnements informatiques, Études de Linguistique Appliquée, pp. 159-171.

Klem, A. M. et Connell, J. P. (2004). Relationships matter: Linking teacher support to student engagement and achievement. The Journal of School Health. 74(7), 262- 273.

Kounin, J.S. (1970). Discipline and group management in classrooms. New York (NY): Holt, Rinehart and Winston. 
Lapointe, J. M. et Legault, F. (2004). Les relations avec l'entourage et l'adaptation psychosociale à l'école secondaire. Canadian Journal of Behavioural Science.36(3), 244-254.

Lessard, A. ; Schmidt, S. (2011). Recension des écrits sur la Gestion de Classe. Université de Sherbrooke

Le français dans le monde, $\mathrm{n}^{\circ}$ spécial (2009). « La perspective actionnelle et l'approche par les tâches en classe de langue ». Clé International, Paris. Leinhardt, G., Weidman, C. et Hammond, K.M. (1987). Introduction and Integration of Classroom Routines by Expert Teachers. Curriculum Inquiry, 17(2), 135-176.

Martineau, S., Gauthier, C. et Desbiens, J.-F. (1999). La gestion de classe au cœur de l'effet enseignant. Revue des sciences de l'éducation. 25(3), 467-496.

McQueen,T (1992). Essentials of classroom management and discipline. New York (NY): Harper Collins.

Muller, C. (2001). The role of caring in the teacher-student relationship for at-risk students. Sociological Inquiry. 71(2), 241-255.

Murdock, T. B., and Miller, A. (2003). Teachers as sources of middle school students' motivational identity: variable-centered and personcentered analytic approaches. The Elementary School Journal. 103(4), 383-399.

Narcy-Combes, J-P., Walski, J. (2004).Le concept de tâche soumis au crible de nouvelles questions. Les Cahiers de l'APLIUT, vol XXIII, pp. 27-44.

Nault, T (1993).Etude exploratoire de l'insertion professionnelle des enseignants débutants au niveau secondaire. Thèse de doctorat. Montréal : Université de Montréal.

Nault, T (1998). L'enseignant et la gestion de classe. Montréal: Les Éditions Logiques.

Nault, T (1999). Eclosion d'un moi professionnel personnalisé et modalités de préparation aux premières rencontres d'une classe. In J.-C. Hétu, M. Lavoie et S. Baillauquès (dir.), Jeunes enseignants et insertion professionnelle (p. 139-161). Bruxelles: De Boeck Université.

Nault, T. (1997). La gestion de classe et ses spécificités. In Comité d'organisation de l'A.E.C.S.E. (dir.), Les actes du Deuxième congrès d'actualité de la recherche en éducation et formation (p. 307-310). France : Nanterre.

Nunan, D. (1989). Designing Tasks for the Communicative Classroom. Cambridge : Cambridge University Press. 
Overton, T. (2004). Promoting academic success through environmental assessment. Intervention in School and Clinic. 39(3), 147-153.

Pêcheur, J. (2010). Rôle des interactions dans l'approche actionnelle, présenté le 24/10/2010 au 7e Congrès Panhellénique et International des Professeurs de Français, Athènes.

Puren, C. (2007).Enseigner et apprendre les langues sur un mode qui change avec le monde: L'exemple du passage de l'approche communicative à la perspective actionnelle ». Seminario Nazionale LEND Bologna 18-19-20

Puren, C. (2009).Conclusion-synthèse : variation sur la perspective de l'agir social en didactique des langues-cultures étrangères. Le français dans le monde, $\mathrm{n}^{\circ}$ spécial « La perspective actionnelle et l'approche par les tâches en classe de langue », pp. 154-168.

Puren, C. (2006).De l'approche communicative à la perspective actionnelle, Le français dans le monde, 347, 37-40.

Puren, C. (2006).La perspective actionnelle : vers une nouvelle cohérence didactique, Le français dans le monde, 348, 42-44.

Reynolds, A. (1995). The Knowledge Base for Beginning Teachers: Education Professionals' Expectations versus Research Findings on Learning to Teach. The Elementary School Journal. 95(3), 199-221.

Richardson, V. et Fallona, C. (2001). Classroom management as method and manner. Journal of Curriculum Studies. 33(6), 705-728.

Robert, J-P. (2008). Dictionnaire pratique de didactique du FLE. Ophrys, Paris.

Rosen, É. (2009). La perspective actionnelle et l'approche par la tâche. Le français dans le monde, $\mathrm{n}^{\circ}$ spécial « La perspective actionnelle et l'approche par les tâches en classe de langue », pp. 6-14.

Rosenberg, M. S. et Jackman, L. A. (2003). Development, implementation, and sustainability of comprehensive school-wide behavior management systems. Intervention in School and Clinic. 39(1), 10-21.

Ryan, A. M. et Patrick, H. (2001). The social environment and changes in adolescents'motivation and engagement during middle school. American Educational Research Journal. 38(2), 437-460.

Sigler, E. A. et Aamidor, S. (2005). From positive reinforcement to positive behaviors: An everyday guide for the practitioner. Early Childhood Educational Journal. 32(4), 249-253.

Smith, S. W. et Gilles, D. L. (2003). Using key instructional elements to systematically promote social skill generalization for students with challenging behavior. Intervention in School and Clinic. 39(1), 30-37. 
Unité proposée basée sur l'approche actionnelle pour le développement de quelques compétences professionnelles

Dr. Manal Mounir Abbas

Springer, C. (2009). La dimension sociale dans le CECR : pistes pour scénariser, évaluer et valoriser l'apprentissage collaboratif. Le français dans le monde, $\mathrm{n}^{\circ}$ spécial «La perspective actionnelle et l'approche par les tâches en classe de langue », pp. 25-35.

Struyk, L.R. (1991). Self-Evaluation Procedures for Teachers. Document ERIC ED336361.

Willis, J. (1996). A Framework for Task-Based Learning. Harlow: Longman.

Wolfgang, CH. (1999). Solving discipline problems. Boston: Allyn and Bacon.

Yinger, R. (1979). Routines in teacher planning. Theory Into Practice, XVIII(3), 163-169.

Yorke, D.B. (1998). Norm setting: Rules by and for the students. Vocational Education Journal, 63(5), 32, 33 et 47. 